\title{
PROCESSAMENTO DE LINGUAGEM NATURAL EM SEGURANÇA E MEIO AMBIENTE: UMA REVISÃO DE LITERATURA
}

\author{
Rodrigo Petrus Domingues (UNIVERSIDADE FEDERAL FLUMINENSE) \\ rodrigo002@gmail.com \\ Valdecy Pereira (UNIVERSIDADE FEDERAL FLUMINENSE) valdecy.pereira@gmail.com \\ Douglas Moreira (UNIVERSIDADE FEDERAL FLUMINENSE) \\ douglas.moreira@europe.com \\ Verônica de Miranda Prottes (UNIVERSIDADE FEDERAL FLUMINENSE) \\ veronicaprottes@hotmail.com
}

\section{Resumo}

Em virtude das lacunas existentes nas políticas de segurança ao longo das últimas décadas, o índice de acidentes nas empresas ao redor do mundo manteve-se elevado implicando em perdas humanas e ambientais sem mensuração. Por outro lado, a facilidade de acesso de informações não estruturadas em formato digital, em conjunto com o desenvolvimento de algoritmos de Inteligência Artificial, tais como o Processamento de Linguagem Natural (PLN), permitiu sistematizar a análise de um volume substancial de informações tirando vantagem do grande poder de processamento dos computadores atuais. Desta forma, este trabalho realizou uma breve revisão de literatura sobre área de Segurança, Meio Ambiente e Saúde (SMS), em conjunto com as ferramentas de PLN e como estas vem sendo empregadas de acordo com o estado da arte. Neste artigo, foi proposto um método para realizar a revisão de literatura deste tema através de PLN, com o desenvolvimento de algoritmo capaz de separar diferentes artigos (dentre um universo de 300 artigos) em agrupamentos semânticos através de técnicas de word embeddings (doc2vec). Em seguida, vinte artigos foram selecionados - amostragem por conveniência e relevância - para análise, permitindo assim identificar lacunas para mitigar questões referentes ao SMS. Por fim, a metodologia apresentou resultados satisfatórios, contudo são extremamente dependentes da quantidade e qualidade dos dados, destacando-se ainda que tanto a utilização quanto a acurácia destes modelos aumentou significativamente na análise de fatores de Segurança, Meio Ambiente e Saúde, à medida em que novas técnicas (ou combinações) destas são criadas e implementadas.

Palavras Chave: Processamento de Linguagem Natural, Segurança, Meio Ambiente e Saúde, Inteligência Artificial, Revisão de Literatura.

\section{Introdução}

A prevenção de acidentes tem como um de seus pilares a necessidade de se aprender com eventos passados, analisando como e por quê ocorreram, evitando assim que se repitam. No entanto, sem um padrão de armazenamento e análise desses eventos, torna-se muito mais difícil extrair informações dos acontecimentos para se aprender com eles. Por conta do baixo desempenho em segurança observado ao longo das últimas décadas, o indice de acidentes nas empresas ao redor do mundo permaneceu elevado, o que implica em perdas humanas e 
ambientais, além de danos à propriedade, muitas vezes irreparáveis. Ao pensar sobre este impacto em termos de sustentabilidade e os novos padrões de produção emergentes nos últimos anos - os quais buscam a utilização ética da força de trabalho, bem como sua saúde efetiva - as tecnologias que englobam o conceito de inteligência artificial surgem como mais uma alternativa no intuito de mitigar os impactos referentes às questões de segurança e meio ambiente, tanto nas firmas quanto na sociedade.

Adicionalmente, testemunhou-se o crescente aumento de dados não estruturados em formato digital nos ultimos anos, fazendo com que as empresas reconhecessem a importância econômica e o poder que esses dados possuem no processo de tomada de decisões gerencial (Ittoo, Nguyen, Van den Bosch, 2015). Caso as bases de dados semi estruturados ou não estruturados sejam corretamente organizadas e analisadas, constituem-se em ferramentas valiosas no ganho em criação de valor para empresas (McKinsey Global Institute, 2015), algo não vislumbrado até anos recentes.

Este trabalho, portanto, apresenta uma breve revisão de literatura sobre como as ferramentas de Inteligência Artificial estão sendo empregadas de acordo com o estado da arte na área de Segurança, Meio Ambiente e Saúde (SMS). Em termos gerais, a área de SMS de uma empresa trabalha com a criação de relatórios de incidentes e acidentes, cujos textos elaborados estruturam os eventos em termos dos fatos ocorridos (por exemplo, quando e como ocorreram, e qual foi a relação de causalidade existente para cada evento). Para a catalogação, classificação e análise destes eventos, com o objetivo de realizar posterior análise de correlação entre eventos e severidade, técnicas como data mining, text mining e processamento de linguagem natural (PLN) podem ser métodos cruciais neste processo. Este trabalho irá se focar sobre o PLN para o processamento destes dados.

O processamento de linguagem natural (PLN) estuda informações não estruturadas produzidas por pessoas, permitindo que computadores possam compreender e analisar o conteúdo descrito de tais mensagens, extraindo informações adicionais através do sentido e significação das palavras. A grande contribuição deste área em evolução está no poder em processar grande quantidade de informação disponibilizada digitalmente. O PLN é a 
confluência de áreas tais como inteligência artificial, linguística e ciência da computação para a produção de sentido da linguagem natural humana. Atualmente, existem alguns instrumentos que permitem essa codificação, como o reconhecimento de voz, tradução de máquina e análise de conteúdo automatizado (Tixier et. al., 2015), resultantes de avanços de pesquisa significativos nos últimos anos.

Desta maneira, empresas com um grande número de empregados ou atividades na qual os trabalhadores estão expostos a um elevado grau de risco necessitam de um grande uso de recursos - sejam eles temporais ou de mão de obra altamente qualificada - para realizar a análise aprofundada de todas as informações que emergem em relatórios. Sem dúvida, trata-se de uma atividade de alto custo financeiro e sem qualquer garantia de eficácia, caso sejam utilizados os métodos tradicionais. Diversos trabalhos científicos apresentam a utilização de métodos de inteligência artificial para detecção de padrões em acidentes como um auxílio ao processamento dos dados (Tixier et. al, 2016; Tixier et. al, 2016b; Fan e Li, 2012; Kuhn, 2018; Nakata, 2017), aumentando a eficiência e eficácia tanto no processo quanto nas informações extraídas. Empresas e a sociedade em geral podem encontrar vantagens ao empregar esses métodos.

Assim, o objetivo deste artigo é fazer uma revisão de literatura sobre a área, apreciando o atual estado da arte da aplicação do processamento de linguagem natural (PLN) para a obtenção de dados em segurança e meio ambiente nas empresas e avaliando as lacunas ainda existentes, seja em relação à aplicação da metodologia, seja em relação as possibilidades ainda não vislumbradas ou não atingidas. Desta maneira, este trabalho pretende contribuir ao lançar luz sobre a importância do tema e sobre recomendações para pesquisas futuras na área. Ressalta-se que neste trabalho foi utilizada a metodologia de Processamento de Linguagem Natural para realizar a seleção dos artigos que compõem esta revisão de literatura, bem como o tema abordado da pesquisa.

O artigo será dividido em quatro etapas, sendo a primeira esta introdução. O tópico seguinte trata da metodologia utilizada para a revisão de literatura acerca do tema (fluxo de operação e 
algoritmos); o tópico 3 apresenta os resultados encontrados no levantamento, e o tópico 4 apresenta as conclusões deste trabalho.

\section{Metodologia}

Este trabalho propõe um método inovador para realizar a revisão de literatura através do processamento natural de linguagem (PNL), com o desenvolvimento de novo software de programação capaz de separar diferentes artigos em agrupamentos semânticos através de técnicas de word embeddings (doc2vec).

\subsection{Extração de arquivo bibtex}

Para extrair as informações bibliográficas (título e resumo), utilizou-se o pacote biblio (Python) que transforma arquivos bibtex (formato padrão para informações bibliográficas) em objetos de programação na linguagem Python.

\subsection{Pré-processamento}

Antes de utilizar o algoritmo doc2vec é necessário - ou em outros casos, recomendável realizar tarefas para tratar o texto, usualmente nesta ordem (Liu; Chiroma; Cocea, 2018):

1. Retirar pontuação e colocar todas as letras em minúsculas;

2. Retirar palavras de ligação (exemplo em Português: e, ou, para etc);

3. Substituir as palavras pelos seus respectivos radicais;

4. Individualizá-las numa lista, mantendo a mesma ordem em que vieram.

\subsection{Word2Vec}

Publicado em 2013 pela Google, word2vec é um algoritmo de código livre que tem o objetivo de representar numa rede neural a representação distribuída das palavras da linguagem natural (Shuai et al., 2018). Existem duas arquiteturas com objetivo de resolver diferentes problemas: 


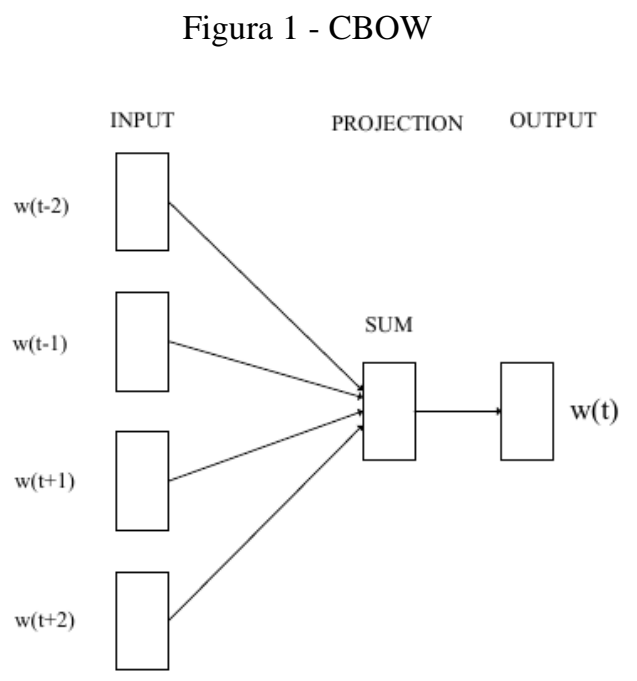

Fonte: Mikolov et. al. (2013)

O Continuous Bag-of-Words (CboW), na Figura 1, prevê a atual palavra através das palavras adjacentes. O skip-gram é utilizado para prever a probabilidade das palavras adjacentes dada uma palavra específica (MIKOLOV et al., 2013). Pode-se concluir com base nos modelos que o CboW tem o potencial de fazer representações melhores com relação as palavras mais comuns, porque ele aloca os vetores considerando o peso das palavras adjacentes e o skipgram, que utiliza a própria palavra para prever os outros vetores e, por isto, representa melhor palavras mais específicas. 
Figura 2 - Skip-gram

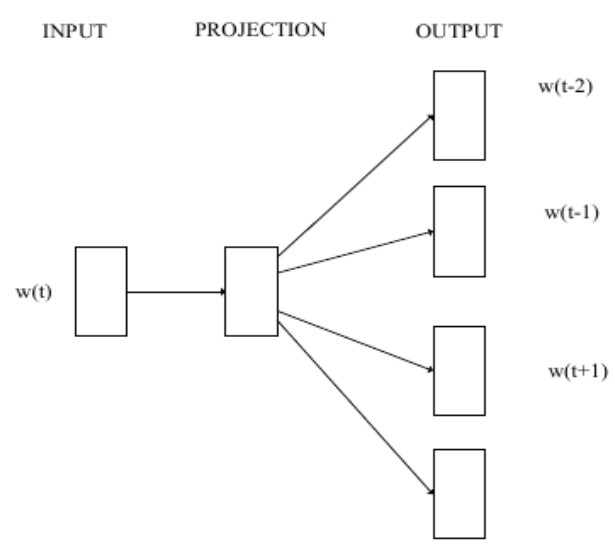

Fonte: Mikolov et. al. (2013)

A literatura indica que devem ser assumidos entre 100 a 1000 vetores para o Word2vec. Para este modelo foram considerados 300 vetores.

\subsection{Doc2vec (também conhecido como Paragraph2vec)}

Baseado no Word2vec, este modelo foi criado para não se perder a ordenação das palavras num documentos, também contendo duas arquiteturas análogas ao Word2vec: Distributed Memory Model of Paragraph Vectors (PV-DM) e Distributed Bag of Words version of Paragraph Vectors (PV- DBOW):

Figura 3 - PV-DM

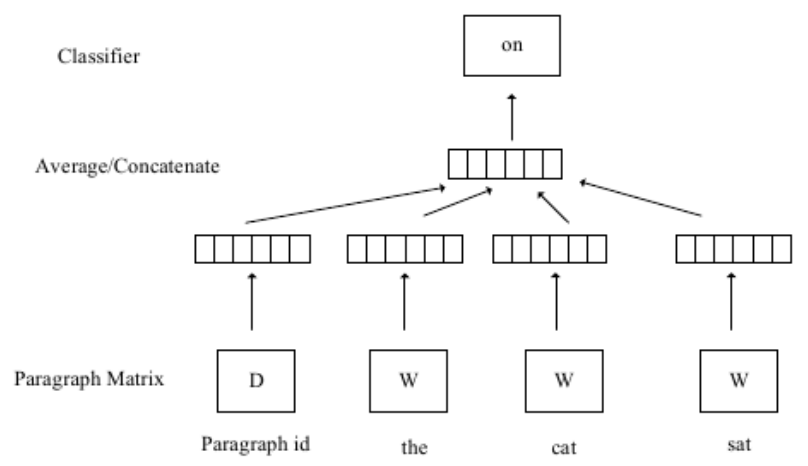

Fonte: Le e Mikolov (2014) 
Figura 4 - PV-DBOW

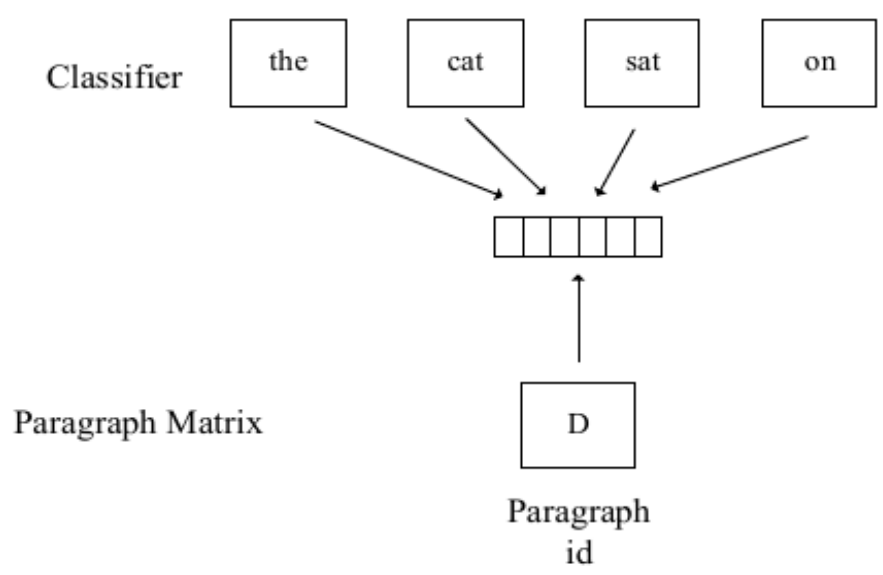

Fonte: Le e Mikolov (2014)

Buscando maximizar a acurácia do modelo e encontrar agrupamentos com afinidade semântica maior, a arquitetura PV-DBOW foi escolhida, devido a sua capacidade de extrair o agrupamento de palavras de maior incidência. Assim sendo, foram considerados como parâmetros uma janela de palavras como 3, taxa de aprendizado à 0,025 com decaimento de 0,00025 por iteração e um total de 100 iterações.

Para a definição do número mínimo de repetições da palavra ao longo do corpus, utilizou-se o princípio de Pareto com o intuito de reduzir a comparação ao menor grupo de palavras que representa aproximadamente $80 \%$ do corpus, como a regra 80/20 de Pareto (AB TALIB; ABDUL HAMID; THOO, 2015).). Esta premissa tem o objetivo de facilitar ao algoritmo a identificação das similaridades semânticas e de relacionamentos de palavras entre os textos.

\subsection{K-Means e medidas de similaridade}

Após a vetorização dos títulos e resumos em 300 (trezentos) vetores, o algoritmo K-Means foi utilizado para realizar a segmentação das publicações, calculando a similaridade pela distância dos cossenos. A escolha destes algoritmos e métodos está fundamentada na revisão de litetatura realizada neste trabalho. Tais abordagens são brevemente apresentadas formalmente, apenas para fins de demonstração dos conceitos propostos nesta metodologia. 
Considere um conjunto de observações $\left(x_{1}, x_{2}, x_{3}, \ldots, x_{n}\right)$ onde cada observaçao $x_{i}$ é um vetor d-dimensional real. Desta forma, o método de agrupamento por K-Means, busca particionar o conjunto de dados de tamanho $\mathrm{n}$ em subconjuntos menores $S=\left\{S_{1}, S_{2}, S_{3}, \ldots, S_{k}\right\}$ limitados a $k(\leq n)$, com o objetivo de minimizar a soma dos quadrados dentro de cada cluster (grupo), ou em outras palavras a variância interna do cluster. Formalmente, o objetivo do K-Means é demonstrado conforme abaixo,

$$
\underset{\mathbf{S}}{\arg \min } \sum_{i=1}^{k} \sum_{\mathbf{x} \in S_{i}}\left\|\mathbf{x}-\boldsymbol{\mu}_{i}\right\|^{2}=\underset{\mathbf{S}}{\arg \min } \sum_{i=1}^{k}\left|S_{i}\right| \operatorname{Var} S_{i}
$$

onde $\mu_{i}$ é a média das observações alocadas dentro do subconjunto $S_{i}$.

Contudo, para medir quão similares são um conjunto de dados (representados por vetores) é mandatório utilizar alguma medida que represente esta semelhança. Conforme mencionado anteriormente, utilizamos a medida de distäncia do cosseno para tal finalidade em virtude desta ser amplamente testada e utilizada em estudos desta natureza.

Desta maneira, considere que o cosseno de dois vetores náo nulos pode ser obtido por meio de um escalar euclidiano da seguinte forma:

$$
\mathbf{A} \cdot \mathbf{B}=\|\mathbf{A}\|\|\mathbf{B}\| \cos \theta
$$

Assim sendo, dados dois vetores de atributos (observações) A e B a similaridade do $\cos \theta$ é representada usando um produtor escalar de magnitude a ser definida pela seguinte equação.

$$
\text { similarity }=\cos (\theta)=\frac{\mathbf{A} \cdot \mathbf{B}}{\|\mathbf{A}\|\|\mathbf{B}\|}=\frac{\sum_{i=1}^{n} A_{i} B_{i}}{\sqrt{\sum_{i=1}^{n} A_{i}^{2}} \sqrt{\sum_{i=1}^{n} B_{i}^{2}}},
$$


Para o 'text matching' (como no caso deste artigo), os vetores de atributos A e B são definidos como os vetores de frequencia de termos dos documentos, variável importante neste trabalho de pesquisa.

Por fim, após esta breve descrição das técnicas de clusterização, particionamento e mensuração de similaridade, os resultados obtidos são apresentados utilizando a metodologia proposta nesta seção.

\section{Resultados}

Consultando a base de publicações Scopus, identificou-se 239 artigos relacionados ao processamento de linguagem natural (PLN) aplicados à segurança, meio ambiente e saúde (SMS).

Como ferramenta, utilizou-se o Natural Language Toolkit (NLTK - Python) para realizar o preprocessamento: foram identificados 3.761 radicais com 29.339 eventos ao longo do corpus. Testou-se a lei de Pareto: $79,9 \%$ do corpus (23.428) é representado por 23,5\% dos radicais (884) mais representativos. O ponto de corte da amostra ficou em 7 eventos do mesmo radical para contabilização ao longo de todo o corpus. Os 20 radicais com o maior número de eventos foram: 'system', 'use', 'safeti', 'languag', 'natur', 'process', 'model', 'requir', 'inform', 'analysi', 'result', 'approach', 'data', 'paper', 'method', 'develop', 'propos', 'control', 'gener', 'base'.

\subsection{Segmentação de vetores}

Utilizou-se o Gensim (Python) para calcular os vetores em doc2vec e o NLTK para a segmentação. Após alguns testes empíricos, divisão ideal de segmentos para este conjunto de publicações foi identificada conforme tabela a seguir (Tabela 1). 
Tabela 1 - Segmentação por publicação.

\section{Grupo}

PLN

Segmentação

Sistemas inteligentes

Otimização

Mineração de dados

Classificação de risco

Interação homem-máquina

Previsão de acidentes

Extração de requisitos

Big data

\section{Publicaçōes}

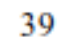

35

27

27

22

21

21

19

18

10

Fonte: Autores.

\subsection{Análise dos resultados}

Com base na segmentação apresentada no tópico anterior, foram analisados os principais trabalhos publicados em cada cluster em termos do número de citações, em virtude de suas principais contribuições e limitações apresentadas, as quais representam ainda desafios no campo em estudo. A tabela (Tabela 2) a seguir apresenta os principais resultados dentre 20 artigos escolhidos pelos autores, respeitando-se a regra de selecionar no minimo um artigo de cada cluster identificado pelo método de K-Means. Ressalta-se ainda, que a seleção dos artigos analisados foi baseada em uma amostragem por conveniência e relevância buscando sintetizar a classificação de cada grupo. Portanto, a Tabela 2 apresenta, para cada trabalho, os autores, tema, contribuições e limitações para pesquisas futuras.

Assim sendo, algumas conclusões podem ser feitas baseando- se nos resultados obtidos. Observou-se que tem havido um investimento intensivo para avaliar em que extensão o PLN e outras ferramentas podem contribuir para o processamento de dados não estruturados de maneira a assegurar a confiabilidade, recuperação e tradução destes em informação que seja valiosa e que apoie a tomada de decisões com vistas a redução de ocorrências e danos. Bons resultados tem sido alcançados até então, uma vez que diversos trabalhos apontam para modelos potencialmente viáveis para aplicação em contextos reais. Esse potencial advém da capacidade de recuperação dos dados coletados em buscas feitas pelos usuários do sistema, pela acurácia dos modelos em traduzir corretamente os dados e pela possibilidade de obtenção 
dos dados a um baixo custo - por exemplo, quando são obtidos em plataformas de mídias sociais.

No entanto, alguns aspectos se colocam como limitações ao uso do PLN, sendo as principais a necessidade de alta quantidade e qualidade de dados disponíveis. Em mídias sociais, por exemplo, a quantidade não é um fator de preocupação, mas a qualidade é afetada por comentários spam e a própria qualidade do texto escrito pelo usuário. Já em contextos empresariais, as limitações podem incluir as duas categorias, já que relatórios de incidentes geralmente podem ser lançados nos sistemas da empresa por qualquer empregado, e não apenas por especialistas no assunto. Em outros trabalhos foi visto que a precisão e a capacidade de recuperação dos dados ainda deixa a desejar devido a um desempenho mediano do modelo. Outras duas limitações que cabem ressaltar são a necessidade de técnicas cada vez mais inovadoras e combinadas ao se tratar de relatórios cuja linguagem e quantidade de termos técnicos seja considerável, como na área da aviação, e a necessidade das empresas se adequarem ao novo modelo pela modificação da maneira como processam os dados de forma manual. 
Quadro 1 - Principais contribuições e limitações dos trabalhos encontrados.

\begin{tabular}{|c|c|c|c|}
\hline Artigo & Resumo & Contribuições & Limitações \\
\hline $\begin{array}{l}\text { Lazzari, M. Salvaneschi, P. Brembilla, L. } \\
\text { (1996) }\end{array}$ & $\begin{array}{l}\text { Utiliza Memória Associativa (por meio de Redes Neurais) para } \\
\text { o Gerenciamento de Segurança Estrutural por meio de uma } \\
\text { descrição qualitativa, o que permite os gerentes de segurança a } \\
\text { interpretar e avaliar as situações de Risco. }\end{array}$ & $\begin{array}{l}\text { Possibilidade de interpretar situações de risco } \\
\text { estrutural (Engenharia Civil) por meio de palavras- } \\
\text { chave estruturadas, um algoritmo e um conjunto de } \\
\text { dados complexo. }\end{array}$ & $\begin{array}{l}\text { Necessidade de se validar o conceito utilizando outras } \\
\text { estruturas de Redes Neurais como RBF (Radial Basis } \\
\text { Function) }\end{array}$ \\
\hline Zadeh, (2007) & $\begin{array}{l}\text { Discute o conceito de Processamento de Linguagem Natural, } \\
\text { como funciona, quais são as limitações e as etapas para } \\
\text { transformar uma informação obtida através de linguagem natura } \\
\text { em um problema com restrições generalizadas que possa ser } \\
\text { tratado computacionalmente. }\end{array}$ & $\begin{array}{l}\text { Os fundamentos apresentados são amplamente } \\
\text { utilizados nos algoritmos de Inteligência Artificial } \\
\text { (Deep Learning) no intuito de calcular probabilidades, } \\
\text { detectar padrões, dentre outras abordagens, utilizando } \\
\text { dados oriundos de linguagem natural. }\end{array}$ & $\begin{array}{l}\text { No momento em que tais técnicas foram apresentadas, } \\
\text { não existia um arcabouço de algorítmos e abordagens } \\
\text { como atualmente. Desta maneira, o artigo não discute } \\
\text { questões operacionais, ficando restrito apenas a } \\
\text { apresentação dos conceitos. }\end{array}$ \\
\hline $\begin{array}{l}\text { Heidarysafa, M. Kowsari, K. Barnes, L. } \\
\text { E., Brown, D. E. (2018) }\end{array}$ & $\begin{array}{l}\text { Analisa um histórico de dados de acidentes em linhas de ferro } \\
\text { nos Estados Unidos para encontrar as causas destes de acordo } \\
\text { com a descrição dos eventos. Como ferramenta, são utilizados } \\
\text { modelos de Processamento de Linguagem Natural e Deep } \\
\text { Learning, a fim de reduzir as inconsistencias dos dados e } \\
\text { fornecendo informações adicionais dos acidentes. }\end{array}$ & $\begin{array}{l}\text { Ao utilizar ferramentas poderosas de Linguagem de } \\
\text { Processamento Natural (como Word2Vec e GloVe) } \\
\text { por meio de Deep Learning, as principais causas de } \\
\text { acidentes nas linhas de ferro no período de quinze } \\
\text { anos foram encontradas, mitigando-se assim as } \\
\text { inconsistências dos relatórios descritivos das } \\
\text { estatísticas de acidentes, extraindo informações úteis } \\
\text { para engenheiros de segurança. }\end{array}$ & $\begin{array}{l}\text { O modelo apresenta maior acurácia em descrições de } \\
\text { acidentes mais frequentes, sendo necessário criar } \\
\text { instrumentos ou alternativas deixar a acurácia mais } \\
\text { homogênea em acidentes de menor incidência. }\end{array}$ \\
\hline $\begin{array}{c}\text { Chokor, A. Naganathan, H. Chong, W. } \\
\text { K. El Asmar, M (2016) }\end{array}$ & $\begin{array}{l}\text { Analisa os dados de acidentes do setor de construção civil do } \\
\text { Estado do Arizona (EUA), por meio de Linguagem de } \\
\text { Processamento Natural e Técnicas de Aprendizado Não } \\
\text { Supervisionado (K-Means). São identificados quatro grupos: } \\
\text { Acidentes Causados por Quedas, Acidentes Causados Objetos } \\
\text { em Queda, Eletrocussões e Desabamentos. }\end{array}$ & $\begin{array}{l}\text { Sugere que o uso de Linguagem de Processamento } \\
\text { Natural é fundamental para que as Agências } \\
\text { Estaduais de Segurança do Trabalho possam criar } \\
\text { normas e classificações de acidentes adequadas a sua } \\
\text { realidade, evitando-se assim a elaboração de normas } \\
\text { sem efeito ou de classificações de acidentes } \\
\text { inadequadas. }\end{array}$ & $\begin{array}{l}\text { O tamanho da amostra utilizado no modelo é limitado } \\
\text { (513 observações) sendo esta uma das justificativas para } \\
\text { se utilizar aprendizado não supervisionado. Também } \\
\text { existe a limitação geográfica da amostra (Estado do } \\
\text { Arizona-EUA). }\end{array}$ \\
\hline $\begin{array}{l}\text { Tixier, A. J.P. Hallowell, M. R. } \\
\text { Rajagopalan, B. Bowman, D. (2016) }\end{array}$ & $\begin{array}{l}\text { Analisa a segurança no ambiente de construção, por meio de } \\
\text { Linguagem de Processamento Natural, em conjunto com } \\
\text { técnicas de aprendizado não supervisionado (Random Forests e } \\
\text { Stochastic Gradient Tree Boosting). De acordo com os autores, } \\
\text { o modelo proposto superou os resultados obtidos na revisão da } \\
\text { literatura referente ao tema, apresentando alta acurácia qual o } \\
\text { tipo de lesão e a parte do corpo atingida de acordo com a } \\
\text { descrição do acidente. }\end{array}$ & $\begin{array}{l}\text { Este trabalho desenvolve uma nova abordagem para a } \\
\text { segurança no ambiente de trabalho por meio de } \\
\text { Linguagem de Processamento Natural, sugerindo o } \\
\text { desenvolvimento de uma nova área de pesquisa em } \\
\text { virtude dos resultados probabilísticos confiáveis. }\end{array}$ & $\begin{array}{l}\text { O grau de predição extremamente baixo para a } \\
\text { severidade da lesão associada ao acidente, tornou-se } \\
\text { uma limitação. Desta maneira, este resultado foi } \\
\text { associado a maneira como os dados estavam } \\
\text { estruturados (de maneira binária e não contínua) o que } \\
\text { inviabilizou a mensuração da severidade do acidente } \\
\text { nestes casos. }\end{array}$ \\
\hline
\end{tabular}




\begin{tabular}{|c|c|c|c|}
\hline Majewski, M. Kacalak, (2006) & $\begin{array}{l}\text { Propoe um modelo inteligente de dupla verificação a fim de } \\
\text { aumentar a segurança de dispositivos por meio de Redes } \\
\text { Neurais. Este modelo propõe através de reconhecimento de voz } \\
\text { e linguagem de processamento natural, na interação homem- } \\
\text { máquina discutindo questões gerais. }\end{array}$ & $\begin{array}{l}\text { Apresenta um fluxo de operação teórico permitindo a } \\
\text { interação homem-maquina por meio de comandos de } \\
\text { voz, detalhando superficialmente como a informação } \\
\text { seria processada pelo dispositivo e quais seriam as } \\
\text { ações tomadas pela máquina diante das instruções } \\
\text { dadas pelo operador. }\end{array}$ & $\begin{array}{l}\text { No momento em que tais técnicas foram apresentadas, } \\
\text { não existia um arcabouço de algorítmos e abordagens } \\
\text { que permitiriam a materialização destes conceitos. }\end{array}$ \\
\hline $\begin{array}{c}\text { Dasgupta, T. Naskar, A. Saha, R. Dey, } \\
\text { L. (2018) }\end{array}$ & $\begin{array}{l}\text { Analisa por meio de Linguagem de Processamento Natural e } \\
\text { Deep Learning um conjunto de informações públicas } \\
\text { disponibilizadas na open web, contendo } 5400 \text { artigos que tratam } \\
\text { de saúde e segurança ocupacional. }\end{array}$ & $\begin{array}{l}\text { O produto final deste estudo trata-se de um registro } \\
\text { contendo os incidentes de segurança em horizontes de } \\
\text { tempo e locais distintos, fornecendo-se assim } \\
\text { informações valiosas para setores da indústria com } \\
\text { alta frequência e severidade de acidentes, permitindo } \\
\text { assim criar normas ou instrumentos para se mitigar } \\
\text { tais riscos. }\end{array}$ & $\begin{array}{l}\text { Apesar das taxas de acurácia serem sempre superiores } \\
\text { a } 65 \% \text {,estes valores podem não ser satisfatórios bem } \\
\text { como não foram tratados problemas de modelagem } \\
\text { como o overfitting. }\end{array}$ \\
\hline $\begin{array}{c}\text { Wu, C. Jiang,P. Ding,C. Feng, F. Chen, T } \\
\text { (2019) }\end{array}$ & $\begin{array}{l}\text { Este artigo se concentra no desenvolvimento de uma rede neural } \\
\text { convolucional para aprender recursos diretamente dos sinais de } \\
\text { vibração originais e diagnosticar falhas em dispositivos } \\
\text { mecânicos rotatórios. }\end{array}$ & $\begin{array}{l}\text { Comparado com os outros três métodos tradicionais, } \\
\text { os resultados mostram que o modelo de rede neural } \\
\text { convolucional unidimensional tem maior precisão para } \\
\text { diagnósticos de falhas de caixa de engrenagens de } \\
\text { eixo fixo e caixa de velocidades planetária do que os } \\
\text { de diagnósticos tradicionais. }\end{array}$ & $\begin{array}{l}\text { Apesar do modelo proposto apresentar alta acurácia } \\
99,3 \% \text {, os autores enfatizam que o uso de redes neurais } \\
\text { convolucionais para identificação de falhas em } \\
\text { dispositivos rotatórios não é um campo de pesquisa } \\
\text { maduro, fazendo com que não exista uma técnica } \\
\text { específica para solucionar tal problema. }\end{array}$ \\
\hline Wu, P. H. Na, P. J. Khoo, C.S.G. (2004) & $\begin{array}{l}\text { O artigo discute duas abordagens principais para a } \\
\text { correspondência de nomes difusos - a abordagem de } \\
\text { processamento de linguagem natural (NLP) e a abordagem de } \\
\text { recuperação de informações (IR) - e propõe uma abordagem } \\
\text { híbrida. }\end{array}$ & $\begin{array}{l}\text { Dois princípios, identidade de atributos de posição } \\
\text { (PAI) e probabilidade de transição de posição (PTL), } \\
\text { são propostos para incorporar aspectos de ambas as } \\
\text { abordagens. Os dois princípios foram implementados } \\
\text { em um sistema de modelo hibrido de PNL e IR } \\
\text { chamado Friendly Name Search (FNS) para } \\
\text { aplicações do mundo real. }\end{array}$ & $\begin{array}{l}\text { Atualmente, Técnicas de Deep Learning poderiam } \\
\text { preencher esta lacuna uma vez que pesquisas recentes } \\
\text { utilizaram tais modelos apresentando acurácia } \\
\text { satisfatória. Contudo, tais técnicas passaram a ser } \\
\text { amplamente utilizadas a partir de 2010, antes da } \\
\text { elaboração deste artigo. }\end{array}$ \\
\hline $\begin{array}{l}\text { Hanif, M.A. Khalid, F. Putra,R. V. W, } \\
\text { Rehman, S. Shafique, M. (2018) }\end{array}$ & $\begin{array}{l}\text { Neste artigo, é apresentada uma visão geral dos desafios } \\
\text { enfrentados para garantir a execução confiável e segura das } \\
\text { Redes Neurais de Camadas Profundas (Deep Learning), bem } \\
\text { como busca demonstrar a importancia de se construir sistemas } \\
\text { robustos de aprendizado de máquina e arquiteturas de hardware } \\
\text { que possam gerar resultados confiáveis na presença de falhas no } \\
\text { nível do hardware, preservando a segurança. }\end{array}$ & $\begin{array}{l}\text { As maiores contribuições deste trabalho foi } \\
\text { apresentar quais eram os maiores desafios em se } \\
\text { construir um hardware seguro que são dedicados a } \\
\text { aplicações de aprendizado de máquina, apresentando } \\
\text { técnicas de ponta que garantam a robustez de tais } \\
\text { modelos, e por fim, os autores apresentam uma } \\
\text { formulação teórica que garantiria a robustez de tais } \\
\text { modelos em um hardware dedicado a tais algorítmos. }\end{array}$ & $\begin{array}{l}\text { O artigo apresenta resultados baseados em um conjunto } \\
\text { de dados simples (base MNIST). Assim sendo, as } \\
\text { medidas propostas para aumentar a robustez do modelo } \\
\text { podem ser seriamente comprometidas, inviabilizando o } \\
\text { aumento da segurança no processamento da informação, } \\
\text { objeto principal deste artigo. }\end{array}$ \\
\hline
\end{tabular}




\begin{tabular}{|c|c|c|c|}
\hline Bian, J., Topaloglu, U., \& Yu, F. (2012). & $\begin{array}{l}\text { Utilizou o Twitter como fonte primária de informações } \\
\text { relacionadas aos efeitos colaterais de medicamentos. A ideia é } \\
\text { fazer controle farmacológico em tempo real dos sintomas. Utiliza } \\
\text { PLN como fonte de obtenção dos dados em um computador de } \\
\text { alta performance. }\end{array}$ & $\begin{array}{l}\text { Possibilidade de obtenção de grande quantidade de } \\
\text { dados e acompanhamento em tempo real dos } \\
\text { sintomas causados por medicamentos }\end{array}$ & $\begin{array}{l}\text { Performance dos modelos de classificação é prejudicada } \\
\text { pelo alto nível de ruído da rede social. }\end{array}$ \\
\hline $\begin{array}{l}\text { Sari, Y., Hassan, M. F., \& Zamin, N. } \\
\text { (2009 }\end{array}$ & $\begin{array}{l}\text { Buscou otimizar o desempenho de NER (Name Entity } \\
\text { Recognition) através de PLN para reconhecimento de categorias } \\
\text { similares em relatórios de segurança e meio ambiente. }\end{array}$ & $\begin{array}{l}\text { Espera-se que com a abordagem de aprendizagem } \\
\text { semi-supervisionada o algoritmo irá criar um léxico } \\
\text { semântico e melhoria a eficiência e acurácia do } \\
\text { sistema. }\end{array}$ & $\begin{array}{l}\text { Não apresentou resultados quantificáveis do trabalho } \\
\text { desempenhado. }\end{array}$ \\
\hline $\begin{array}{l}\text { Stocker, C., Marzi, L.-M., Matula, C., } \\
\text { Schantl, J., Prohaska, G., Brabenetz, A., } \\
\text { \& Holzinger, A. (2014). }\end{array}$ & $\begin{array}{l}\text { Buscou realizar a extração de dados de relatórios médicos } \\
\text { cirúrgicos através de PLN com o objetivo de melhoria para a } \\
\text { segurança do paciente no contexto hospitalar. }\end{array}$ & $\begin{array}{l}\text { A abordagem de frases desencadeadas parece muito } \\
\text { promissora à medida em que abre a opção para lidar } \\
\text { com dados não vistos. }\end{array}$ & $\begin{array}{l}\text { A maior restrição no design do projeto é a falta de } \\
\text { recursos ontológicos disponíveis publicamente }- \text { os } \\
\text { relatórios utilizados estavam em alemão. }\end{array}$ \\
\hline $\begin{array}{c}\text { Tixier, A. J.-P., Hallowell, M. R., } \\
\text { Rajagopalan, B., \& Bowman, D. (2016). }\end{array}$ & $\begin{array}{l}\text { O artigo descreve o uso de PLN para o levantamento de } \\
\text { informações em relatórios de segurança da área de construção } \\
\text { civil, como o sistema foi construído e as implicações e potencial } \\
\text { para o uso dos dados extraídos. }\end{array}$ & $\begin{array}{l}\text { O PLN é capaz de levantar dados não estruturados } \\
\text { de lesões rápida e automaticamente para relatórios de } \\
101 \text { atributos e saídas com mais de } 95 \% \text { de acurácia. }\end{array}$ & $\begin{array}{l}\text { O sistema não pode abordar situações que não foram } \\
\text { antecipadas, e a qualidade de dados textuais disponíveis } \\
\text { afeta diretamente a qualidade dos atributos e dados de } \\
\text { saída extraídos pelo sistema. }\end{array}$ \\
\hline $\begin{array}{l}\text { Tanguy, L., Tulechki, N., Urieli, A., } \\
\text { Hermann, E., \& Raynal, C. (2016). }\end{array}$ & $\begin{array}{l}\text { Busca descrever as diferentes técnicas de PLN utilizadas e } \\
\text { usadas em colaboração entre empresas de pesquisa de dados e } \\
\text { laboratórios de pesquisa para a extração de dados de relatórios } \\
\text { de anormalidades em voos de aviação civil. }\end{array}$ & $\begin{array}{l}\text { Essas anormalidades podem apontar para a presença } \\
\text { de perigos existentes e são fonte útil de } \\
\text { investigação.PLN é uma maneira eficaz de organizar } \\
\text { e padronizar os dados devido a sua grande variedade } \\
\text { e número. }\end{array}$ & $\begin{array}{l}\text { O PLN não pode ser utilizado de maneira direta nesses } \\
\text { casos; é necessário o uso de técnicas inovadoras para } \\
\text { manipular as especificidades dos dados da área de } \\
\text { aviação. }\end{array}$ \\
\hline $\begin{array}{c}\text { Rosadini, B., Ferrari, A., Gori, G., } \\
\text { Fantechi, A., Gnesi, S., Trotta, I., \& } \\
\text { Bacherini, S. (2017). }\end{array}$ & $\begin{array}{l}\text { Buscou investigar em que medida o PLN pode ser aplicada de } \\
\text { forma prática para detectar defeitos nos documentos de } \\
\text { requisitos de um fabricante de sinalização ferroviária, uma vez } \\
\text { que a sinalização de requisitos de qualidade pode ser feita em } \\
\text { linguagem natural, dentro de documentos com requisitos. }\end{array}$ & $\begin{array}{l}\text { O estudo trouxe uma comparação entre as técnicas } \\
\text { manuais tradicionais usadas na indústria para análise } \\
\text { de requisitos e a análise desempenhada com o PLN. }\end{array}$ & $\begin{array}{l}\text { As limitações da implementação do PLN surgem das } \\
\text { discrepâncias entre a abordagem de análise manual dos } \\
\text { especialistas e o PLN, sugerindo pistas para melhorar as } \\
\text { capacidades das técnicas de PLN com soluções } \\
\text { específicas para as firmas, e sugere também que as } \\
\text { práticas das empresas precisam ser modificadas para } \\
\text { efetivamente explorar ferramentas PLN. }\end{array}$ \\
\hline
\end{tabular}




\begin{tabular}{|c|c|c|c|}
\hline $\begin{array}{l}\text { Chowdhury, F. B. Abacha, B. A. } \\
\text { Lavelli, A. Zweigenbaum P., (2011) }\end{array}$ & $\begin{array}{l}\text { O trabalho explora a combinação de duas abordagens diferentes } \\
\text { de machine learning para a extração de detecção de interação } \\
\text { entre drogas (DDI) - ou seja, quando uma droga faz efeito de } \\
\text { interação com outras drogas. }\end{array}$ & $\begin{array}{l}\text { A abordagem conjunta das duas propostas se mostra } \\
\text { positiva em termos do aumento da pontuação } \mathrm{F} 1 \text {. }\end{array}$ & $\begin{array}{l}\text { A precisão e a capacidade de recordar ainda são baixas } \\
\text { dentro do que é considerado necessário para um sistema } \\
\text { desse tipo. }\end{array}$ \\
\hline $\begin{array}{l}\text { Isah, H., Trundle, P., \& Neagu, D. } \\
\text { (2014). }\end{array}$ & $\begin{array}{l}\text { Buscou desenvolver uma estrutura para coleta e análise de } \\
\text { dados em redes sociais sobre as visões e sentimentos dos } \\
\text { usuários de medicamentos e produtos cosméticos usando } \\
\text { machine learning, text mining e análise de sentimento. O objetivo } \\
\text { é realizar o acompanhamento de marcas, falsificações e } \\
\text { tendências no sentimento relacionados a produtos lançados no } \\
\text { mercado. }\end{array}$ & \begin{tabular}{|l|} 
Os autores mostram a utilidade da estrutura para \\
realizar acompanhamento de tendências através da \\
análise de sentimentos em comentários do Facebook e \\
Twitter.
\end{tabular} & $\begin{array}{l}\text { A estrutura ainda não considera detecção de } \\
\text { comentários spam, comparação de desempenho entre } \\
\text { abordagens de machine learning para classificação de } \\
\text { sentimentos, detecção de tendências de crescimento ou } \\
\text { diminuição de aceitação de produtos. }\end{array}$ \\
\hline $\begin{array}{c}\text { Daramola, O., Stalhane, T., Sindre, G., \& } \\
\text { Omoronyia, I. (2011) }\end{array}$ & $\begin{array}{l}\text { Apresenta uma estrutura e protótipo da ferramenta KROSA } \\
\text { baseada em PLN que facilita a identificação inicial de potenciais } \\
\text { perigos ao sistemas através de requisitos e de reutilização de } \\
\text { experiência previa ao conduzir uma análise HAZOP. }\end{array}$ & $\begin{array}{l}\text { A ferramenta possui potencial para aplicação em } \\
\text { contextos reais industriais. }\end{array}$ & $\begin{array}{l}\text { O desempenho da ferramenta KROSA depende } \\
\text { significativamente da qualidade e do domínio ontológico, } \\
\text { assim o desempenho pode ficar muito baixo ao depender } \\
\text { de sua qualidade global. }\end{array}$ \\
\hline Kim, T., \& Chi, S. (2019). & $\begin{array}{l}\text { Propõe um sistema de gestão do conhecimento para coleta e } \\
\text { recuperação de casos de acidentes em construção civil usando } \\
\text { PLN. }\end{array}$ & $\begin{array}{l}\text { O sistema possui boa recuperação de resultados que } \\
\text { são } 97 \% \text { relevantes para os casos de acidentes } \\
\text { buscados pelo usuário e também analisar informação } \\
\text { com boa acurácia para dois modelos. }\end{array}$ & $\begin{array}{l}\text { Necessidade de alta quantidade e qualidade de dados } \\
\text { disponíveis; Alta dependência de dicionários e o volume } \\
\text { de informações, ao se tornar maior, deve limitar o } \\
\text { número de casos relevantes a serem utilizados; } \\
\text { Limitações práticas em geração de regras de relatórios } \\
\text { de acidentes originais envolvendo erros gramaticais e } \\
\text { várias expressões. }\end{array}$ \\
\hline
\end{tabular}

Fonte: Autores 


\subsection{Impacto sobre SMS}

Dos trabalhos acima apresentados, pode-se evidenciar pelo menos 4 contribuições que a PLN tem dentro do contexto organizacional em SMS. A primeira diz respeito à detecção de padrões nos dados levantados (LAZZARI, M. SALVANESCHI, P. BREMBILLA, L.1996; ZADEH, 2007; HEIDARYSAFA, M. KOWSARI, K. BARNES, L. E., BROWN, D. E. , 2018). Os padrões geram a possibilidade de interpretar situações de risco, facilitando o cálculo de probabilidades de eventos, auxiliando na consequente gestão de riscos de SMS de uma empresa. Através destes padrões também são mitigadas as inconsistências dos relatórios descritivos das estatísticas de acidentes, extraindo informações úteis para engenheiros de segurança.

A segunda contribuição diz respeito à capacidade do uso de Processamento de Linguagem Natural para a criação de normas e classificações de acidentes adequadas à realidade de uma empresa, evitando-se a elaboração de normas sem efeito ou de classificações de acidentes inadequadas (CHOKOR, A. NAGANATHAN, H. CHONG, W. K. EL ASMAR, M., 2016; DASGUPTA, T. NASKAR, A. SAHA, R. DEY, L., 2018). Esse aspecto é fundamental, pois, na atualidade, a adaptação de métodos e ferramentas para a gestão de riscos de uma empresa por meio de empresas de consultoria ou de benchmarking, se não for bem adaptada à realidade da mesma, produz uma série de procedimentos que não representam a essência dos riscos específicos das atividades da organização.

A terceira contribuição é relativa à maior acurácia para a recuperação de dados relevantes em casos de acidentes (KIM, T., \& CHI, S. , 2019). Tal característica se torna relevante à medida em que permite que o processo de gestão do conhecimento de uma empresa se torne eficaz de maneira mais eficiente, economizando tempo e recursos neste ciclo. No entanto, essa potencial contribuição se torna mais palpável ao lidar com grande quantidade de dados, beneficiando neste sentido as empresas de grande porte.

A última grande contribuição destacada aqui se refere aos trabalhos que demonstraram a capacidade que o PLN tem de extrair padrões de detecção de sentimentos na avaliação de produtos farmacêuticos, e as interações medicamentosas em indivíduos através de simples relato em redes sociais (CHOWDHURY, F. B. ABACHA, B. A. LAVELLI, A. ZWEIGENBAUM P., 2011; ISAH, H., TRUNDLE, P., \& NEAGU, D., 2014). Isso demonstra o potencial que se levanta na avaliação de aspectos subjetivos dos indivíduos, tão importantes dentro do contexto de SMS. 
Essa contribuição pode ser vista de duas formas: por um lado, a capacidade de detectar sentimentos pode auxiliar em momentos cruciais para a realização de atividades de alto risco, como por exemplo, as que envolvam altura, eletricidade, etc., em que a condição de saúde física e emocional/mental do indivíduo deve ser avaliada em tempo real. Associar declaração de sentenças em um sistema de registro com condições de saúde potencialmente perigosas é um benefício real para a interrupção de uma atividade que pode gerar incidentes. Por outro lado, os relatos podem beneficiar o cruzamento de informações entre atividades/sentimentos como uma maneira de se avaliar melhor o aspecto causa-consequência de questões que cercam o ambiente das tarefas dentro de um processo. Em ambos os casos, a gestão de SMS é substancialmente beneficiada.

Os trabalhos aqui apresentados ressaltam o grande potencial que o PLN possui para gerar resultados palpáveis no auxílio à tomada de decisões dentro das empresas. A necessidade crescente de cuidar dos recursos humanos de uma empresa, em termos de sustentabilidade da organização, passa pela segurança no sentido amplo e prevenção aos acidentes no sentido estrito. Neste quesito o PLN pode se tornar um grande aliado por fornecer apoio a decisão, já que o grande volume de informações processadas, em menor tempo e custo, podem evidenciar padrões de ocorrências de acidentes, e a possibilidade de uma análise criteriosa e aprofundada de causaefeito. Tudo isso contribui para a gestão da segurança e do meio ambiente em um ambiente organizacional.

\subsection{Utilidade da ferramenta desenvolvida para realizar a revisão da literatura}

A ferramenta desenvolvida permite o monitoramento constante de novos artigos, estabelecendo segmentos de conhecimento de forma automática. Estes segmentos demonstram diferentes temas relacionados ao SMS, facilitando a identificação de artigos relevantes de acordo com o assunto de interesse. A capacidade da ferramenta em gerar novos agrupamentos permite a identificação de novos campos de conhecimento em nascimento, quais são os artigos mais citados ou com maior impacto em cada tema (em conjunto com as estatísticas bibliográficas) e a tendência de crescimento de um novo campo de estudo. Este acompanhamento baseado em grupos de artigos, em substituição ao levantamento de artigos individualizados, permite visualizar qual o conhecimento de vanguarda que está sendo gerado pelos pesquisadores e estudado de forma coletiva na atualidade ou em períodos específicos. 


\section{Conclusão}

Este trabalho apresentou uma revisão de literatura acerca do uso de Processamento de Linguagem Natural como uma ferramenta para codificação de dados não estruturados ou semiestruturados para aplicação na área de SMS - Segurança, Meio Ambiente e Saúde. Para avaliar a produção a este respeito, uma metodologia de coleta dos dados através de PLN também foi utilizada, com o desenvolvimento de novo software de programação capaz de separar diferentes artigos em agrupamentos semânticos através de técnicas de word embeddings (doc2vec). Após separação dos grupos, foram avaliadas as principais contribuições e limitações dos trabalhos realizados para relacionar as novidades na área e suas possibilidades para a área de SMS.

O PLN é um campo atraente e tem sido palco de investimento intensivo em esforços nos últimos anos. Os resultados mostram-se promissores à medida que reduzem o investimento em custo e recursos humanos para apoio à tomada de decisão. Mas, no âmbito da segurança, saúde e meio ambiente esses benefícios significam também a preservação de vidas, do patrimônio e de sustentabilidade do negócio.

Por outro lado, o fato de ser um domínio ainda não maduro e em constante evolução gera algumas limitações para sua aplicação. Por exemplo, na análise qualitativa dos artigos agrupados de acordo com a metodologia proposta foram identificadas discrepâncias que precisam ser corrigidas como clusters de artigos não homogêneos. A alocação incorreta (mesmo considerando um Índice de Pareto superior a 80\%) de artigos em subgrupos distintos demonstra a necessidade de se fazer um ajuste fino, tanto no número de clusters, como nos parâmetros de entrada do código proposto. Ressalta-se também a dependência da quantidade e qualidade de dados é imperativo, enquanto contextos reais nem sempre fornecem tais condições, como é o caso do ambiente corporativo. No entanto, o potencial para a utilização desses modelos aumenta à medida que novas técnicas inovadoras e combinações delas são criadas e implementadas.

Por fim, para a área de Segurança, Meio Ambiente e Saúde (SMS) o Processamento de Linguagem Natural é mais uma ferramenta, dentro de uma miríade de técnicas já utilizadas com o objetivo de extrair informações adicionais referentes aos acidentes, bem como detectar novos padrões de risco, permitindo a atualização do marco regulatório referente a este tema caro para sociedade moderna. 


\section{REFERÊNCIAS}

AB TALIB, M. S.; ABDUL HAMID, A. B.; THOO, A. C. Critical success factors of supply chain management: a literature survey and Pareto analysis. EuroMed Journal of Business, v. 10, n. 2, p. 234-263, 6 jul. 2015.

BIAN, J.; TOPALOGLU, U.; YU, F. Towards large-scale twitter mining for drug-related adverse events. International Conference on Information and Knowledge Management, Proceedings. Anais...2012Disponível em: <https://www.scopus.com/inward/record.uri?eid=2-s2.0-

84870431352\&doi=10.1145\%2f2389707.2389713\&partnerID=40\&md5=d9f9fbc01035dc468da0764adb92a853>

CHOKOR, A., NAGANATHAN, H., CHONG, W. K., \& ASMAR, M. E. Analyzing Arizona OSHA Injury Reports Using Unsupervised Machine Learning. Procedia Engineering, 145, 1588-1593.doi:10.1016/j.proeng.2016.04.200, 2016.

CHUNZHI W., PENG C.J., CHUANG D., FUZHOU F., TANG C. Intelligent fault diagnosis of rotating machinery based on one-dimensional convolutional neural network, 2019.

DARAMOLA, O., STALHANE, T., SINDRE, G., \& OMORONYIA, I. Enabling hazard identification from requirements and reuse-oriented HAZOP analysis. 2011 4th International Workshop on Managing Requirements Knowledge.doi:10.1109/mark.2011.6046555, 2011.

DASGUPTA, T., NASKAR, A., SAHA, R., \& DEY, L. Extraction and Visualization of Occupational Health and Safety Related Information from Open Web. 2018 IEEE/WIC/ACM International Conference on Web Intelligence (WI).doi:10.1109/wi.2018.00-56, 2018.

HANIF, M. A., KHALID, F., PUTRA, R. V. W., REHMAN, S., \& SHAFIQUE, M. Robust Machine Learning Systems: Reliability and Security for Deep Neural Networks. 2018 IEEE 24th International Symposium on OnLine Testing And Robust System Design (IOLTS).doi:10.1109/iolts.2018.8474192, 2018.

HEIDARYSAFA, M., KOWSARI, K., BARNES, L., BROWN, D. Analysis of Railway Accidents' Narratives Using Deep Learning. 2018 17th IEEE International Conference on Machine Learning and Applications (ICMLA).doi:10.1109/icmla.2018.00235, 2018.

HENRY, S.; CUFFY, C.; MCINNES, B. T. Vector representations of multi-word terms for semantic relatedness. Journal of Biomedical Informatics, v. 77, p. 111-119, jan. 2018.

ISAH, H., TRUNDLE, P., \& NEAGU, D. Social media analysis for product safety using text mining and sentiment analysis. 2014 14th UK Workshop on Computational Intelligence (UKCI).doi:10.1109/ukci.2014.6930158, 2014.

KIM, T., \& CHI, S. Accident Case Retrieval and Analyses: Using Natural Language Processing in the Construction Industry. Journal of Construction Engineering and Management, 145(3), 04019004.doi:10.1061/(asce)co.19437862.0001625, 2019.

LAZZARI, M.; SALVANESCHI, P.; BREMBILLA, L. Looking for analogies in structural safety management through connectionist associative memories. Proceedings of International Workshop on Neural Networks for Identification, Control, Robotics, and Signal/Image Processing, NICROSP. Anais...1996Disponível em: <https://www.scopus.com/inward/record.uri?eid=2-s2.00029726674\&partnerID=40\&md5=b61967a6f0db31dbe058d9c452851138>

LE, Q. V.; MIKOLOV, T. Distributed Representations of Sentences and Documents. arXiv:1405.4053 [cs], 16 maio 2014.

LIU, H.; CHIROMA, F.; COCEA, M. Identification and classification of misogynous tweets using multiclassifier fusion. . In: CEUR WORKSHOP PROCEEDINGS 2150. 2018. 
MAJEWSKI, M.; KACALAK, W. Natural language human-machine interface using artificial neural networks. Lecture Notes in Computer Science (including subseries Lecture Notes in Artificial Intelligence and Lecture Notes in Bioinformatics), v. 3973 LNCS, p. 1161-1166, 2006.

MAJEWSKI, MACIEJ \& KACALAK, WOJCIECH. Automatic Recognition and Evaluation of Natural Language Commands. Lecture Notes in Computer Science. 3973. 1155-1160. 10.1007/11760191_168, 2006.

MIKOLOV, T. et al. Efficient Estimation of Word Representations in Vector Space, 2013.

NAKATA, T. Text-mining on incident reports to find knowledge on industrial safety. Proceedings - Annual Reliability and Maintainability Symposium. Anais...2017Disponível em:

$<$ https://www.scopus.com/inward/record.uri?eid=2-s2.0-

85018618350\&doi=10.1109\%2fRAM.2017.7889795\&partnerID=40\&md5=700011817afc2faac621dfd7e37cb975>

ROSADINI, B., FERRARI, A., GORI, G., FANTECHI, A., GNESI, S., TROTTA, I., \& BACHERINI, S. Using NLP to Detect Requirements Defects: An Industrial Experience in the Railway Domain. Lecture Notes in Computer Science, 344-360. doi:10.1007/978-3-319-54045-0_24, 2017.

SARI, Y., HASSAN, M. F., \& ZAMIN, N. A Hybrid Approach to Semi-supervised Named Entity Recognition in Health, Safety and Environment Reports. 2009 International Conference on Future Computer and Communication.doi:10.1109/icfcc.2009.52, 2009.

SHUAI, Q. et al. Sentiment Analysis on Chinese Hotel Reviews with Doc2Vec and Classifiers. (2018) IEEE 3rd Advanced Information Technology, Electronic and Automation Control Conference (IAEAC). In: 2018 IEEE 3rd Advanced Information Technology, Electronic And Automation Control Conference (IAEAC). Chongqing: IEEE, out. 2018.

STOCKER, C. et al. Enhancing patient safety through human-computer information retrieval on the example of german-speaking surgical reports. Proceedings - International Workshop on Database and Expert Systems Applications, DEXA. Anais...2014Disponível em: <https://www.scopus.com/inward/record.uri?eid=2-s2.084919342925\&doi=10.1109\%2fDEXA.2014.53\&partnerID=40\&md5=33a26ba69bf2e157e4da11d224349624>

TAKENAKA, K., BANDO, T., NAGASAKA, S., TANIGUCHI, T., \& HITOMI, K. Contextual scene segmentation of driving behavior based on double articulation analyzer. 2012 IEEE/RSJ International Conference on Intelligent Robots and Systems.doi:10.1109/iros.2012.6385614, 2012.

TANGUY, L., TULECHKI, N., URIELI, A., HERMANN, E., \& RAYNAL, C. Natural language processing for aviation safety reports: From classification to interactive analysis. Computers in Industry, 78, 8095.doi:10.1016/j.compind.2015.09.005, 2015.

TIXIER, A. J.-P., HALLOWELL, M. R., RAJAGOPALAN, B., \& BOWMAN, D.. Automated content analysis for construction safety: A natural language processing system to extract precursors and outcomes from unstructured injury reports. Automation in Construction, 62, 45-56.doi:10.1016/j.autcon.2015.11.001, 2016.

TIXIER, A. J.-P. et al. Application of machine learning to construction injury prediction. Automation in Construction, v. 69, p. 102-114, 2016a.

WU et al. (2004). NLP Versus IR Approaches to Fuzzy Name Searching in Digital Libraries. Research and Advanced Technology for Digital Libraries, 2004. Springer Berlin Heidelberg, Berlin, Heidelberg, 978-3-54030230-8. 10.1007/978-3-540-30230-8_14.

ZADEH, L. A. A New Frontier in Computation-Computation with Information Described in Natural Language. 2007 IEEE International Symposium on Intelligent Signal Processing.doi:10.1109/wisp.2007.4447531, 2007. 